\title{
Las transformaciones de la enseñanza en los inicios universitarios: una experiencia en el contexto de la virtualidad forzada
}

The transformations of teaching in university beginnings: An experience in the context of forced virtuality

\author{
Fernando Bifano \\ https://orcid.org/0000-0001-5925-5794 \\ fjbifano@gmail.com \\ Instituto de Estudios Iniciales | Universidad \\ Nacional Arturo Jauretche | Argentina
}

\section{Lorena Cabaña}

https://orcid.org/0000-0002-8639-1297

lorena.cabana59@gmail.com

Instituto de Estudios Iniciales | Universidad

Nacional Arturo Jauretche | Argentina

\author{
Alejandra Almirón \\ https://orcid.org/0000-0001-5146-5738 \\ alealmi83@gmail.com \\ Instituto de Estudios Iniciales | Universidad \\ Nacional Arturo Jauretche | Argentina
}

\author{
Karina Gonzalez \\ https://orcid.org/0000-0002-7944-2463 \\ karinafiles@yahoo.com.ar \\ Instituto de Estudios Iniciales | Universidad \\ Nacional Arturo Jauretche | Argentina
}

\section{RESUMEN}

Los inicios universitarios vienen siendo motivo de investigación, reflexión y debate durante las últimas décadas a partir de la masificación del ingreso a la educación superior. En este año tan particular, se suman a esta discusión nuevas preguntas: ¿qué lecciones hemos aprendido del tiempo de enseñanza transcurrido en la virtualidad forzada? (Bifano, Almirón, Cabaña, Gonzalez, 2020) ¿Qué tipo de recursos de los implementados en el contexto de trabajo llevado adelante durante el ASPO permanecerán en una potencial vuelta a la normalidad?

Estas y otras preguntas son las que orientan nuestra investigación llevada a cabo en simultáneo con el proceso de virtualización (Orozco, 2013) que transformó la enseñanza de las materias del ciclo inicial de la Universidad Nacional Arturo Jauretche durante 2020. Siguiendo los aportes de la metodología de la teoría fundamentada (Lora, Cavadias \& Miranda; 2017) daremos cuenta de cómo se vio transformada la enseñanza: ¿qué decisiones tomaron los equipos docentes de las materias?, ¿cómo los espacios virtuales fomentaron el trabajo colectivo docente?, ¿en qué medida se pudieron readaptar prácticas en la virtualidad?, ¿cuáles fueron los principales factores que condicionaron la enseñanza? Son algunos de los interrogantes que nos invitan a la reflexión y nos desafían a pensar las continuidades en el regreso a la presencialidad.

\section{PALABRAS CLAVE}

Virtualidad forzada, Inicios universitarios, Clase virtual, Teoría fundamentada. 


\section{KEY WORDS}

Forced virtuality, University beginnings, Virtual classes,

Grounded Theory.

\section{ABSTRACT}

University beginnings have been a reason for research, reflection and debate during the last decades from the massification of admission to higher education. In this very particular year, new questions are added to this discussion: what lessons have we learned from the teaching time spent in forced virtuality (Authors, 2020)? What type of resources from those implemented in the context of the work carried out during the ASPO will remain in a potential return to normality?

These and other questions are the ones that guide our research carried out simultaneously with the virtualization process (Orozco 2013) that transformed the teaching of the subjects of the initial cycle of the Arturo Jauretche National University during 2020. Following the contributions of the methodology of the grounded theory (Lora, Cavadias \& Miranda; 2017) we will give an account of how teaching was transformed: what decisions did the teaching teams of the subjects make? How did virtual spaces promote collective teaching work? To the extent that virtual practices could be readapted? What were the main factors that conditioned teaching? These are some of the questions that invite us to reflect and challenge us to think about continuities in the return to presence. 


\section{INTRODUCCIÓN}

El proceso de virtualización comprende, según Orozco (2013), una serie de dimensiones que necesitan ser articuladas: lo organizacional, lo pedagógico, lo tecnológico y lo comunicacional. En circunstancias extraordinarias como las que se vivieron a partir de la pandemia del covid-19, dicho proceso se vió vertiginosamente acelerado y en algún sentido, forzado (Bifano, Almirón, Cabaña, Gonzalez, 2020) a tomar como única opción ante la demanda de dar respuestas a la continuidad pedagógica que exigió a la educación en general, y a la comunidad universitaria en particular, adoptar los medios para garantizar el derecho a la educación. En este texto, presentaremos algunos elementos de dicho proceso de toma de decisiones y cómo estas afectaron al ejercicio de la docencia en el marco de los inicios universitarios en la Universidad Nacional Arturo Jauretche (en adelante UNAJ).

La UNAJ cuenta con un instituto dedicado exclusivamente al ingreso universitario y que nuclea cuatro materias que componen el Ciclo Inicial: Matemática, Prácticas Culturales, Problemas de Historia Argentina y el Taller de Lectura y Escritura -en adelante MI, PC; PHA y TLE respectivamente según corresponda-. Está integrado por alrededor de 250 docentes con diversos perfiles de formación que dictan estas materias para un alumnado que en los últimos años ha superado los diez mil inscriptos. El proceso de virtualización forzada implicó, de parte de las coordinaciones de cada materia, la elaboración de materiales para disponer en aulas de moodle donde cada docente tuvo a cargo durante cada cuatrimestre dos comisiones de entre 35 y 45 estudiantes.

El proceso de virtualización forzada implicó, de parte de las coordinaciones de cada materia, la elaboración de materiales para disponer en aulas de moodle donde cada docente tuvo a cargo durante cada cuatrimestre dos comisiones de entre 35 y 45 estudiantes. 


\section{MARCO TEÓRICO-METODOLÓGICO}

Para esta investigación, asumimos el marco teórico-metodológico de la teoría fundamentada, desarrollada por Glaser y Strauss (1999), e integrada al ámbito de la educación por Lora, Cavadias y Miranda (2017). Esta postura nos permitirá construir las categorías analíticas para comprender y analizar cómo se reconfigura el sistema de recursos del profesor (Gueudet y Trouche 2009) al momento de afrontar la planificación y la enseñanza en este contexto.

Para la etapa preliminar de la investigación se envió un formulario con el fin de relevar las expectativas respecto de la experiencia de desarrollar las clases virtuales, que respondieron libremente unos 83 docentes, 50 de los cuales aceptaron ser vueltos a contactar para ser entrevistados. A estos, se les envió, en primer término, a principio del cuatrimestre, un correo electrónico -cf. fig. 1- donde además de compartir con ellos los resultados globales de las respuestas obtenidas al formulario, les solicitamos que nos explicaran, mediante una serie de capturas de pantalla acompañados de un texto o un audio, en qué consistía el trabajo docente en este marco de emergencia.

\footnotetext{
Estimadxs:

Queremos agradecerles su interés por ser nuevamente contactadxs para continuar con la investigación. Ante todo, les queremos compartir los primeros resultados cuantitativos arrojados por las respuestas (en adjunto a este correo).

A la vez, y con la intención de dar entonces continuidad con la investigación, les vamos a
proponer la siguiente actividad. Sabemos que exigirá algo de tiempo y un nuevo compromiso
de su parte, y esperamos que todxs aquellxs a quienes les sea posible, nos puedan enviar su
aporte.
}

Todos somos conscientes de que este escenario resulta totalmente novedoso y por eso queremos poder analizar en qué consiste el trabajo docente en este contexto de emergencia. Les proponemos, concretamente, una serie de capturas de pantallas (las que uds. consideren necesarias) que representan el trabajo que desarrollan dentro del aula virtual de su materia para una clase. Luego, con ellas, armen un texto o graben un audio que explique porqué las eligieron como emblemáticas de este trabajo. No hay un límite de capturas y puede ser cualquier tema que se esté desarrollando.

Una vez hayan completado esto, les pedimos que nos lo envien como respuesta a este correo. Desde ya, les agradecemos una vez más por el compromiso con esta investigación que nos permitirá seguramente poder comprender mejor nuestro trabajo en este nuevo escenario de virtualidad "forzada".

Saludos cordiales

Figura 1: correo electrónico enviado para que nos explicaran mediante capturas de pantalla en qué consistía la tarea docente.

Recibimos pocas pero variadas respuestas: algunos archivos con explicaciones sobre las capturas de pantalla seleccionadas, en otros casos, una presentación multimedial con notas de audio incorporadas a cada diapositiva, entre otras -cf. fig. 2 y 3 -. 


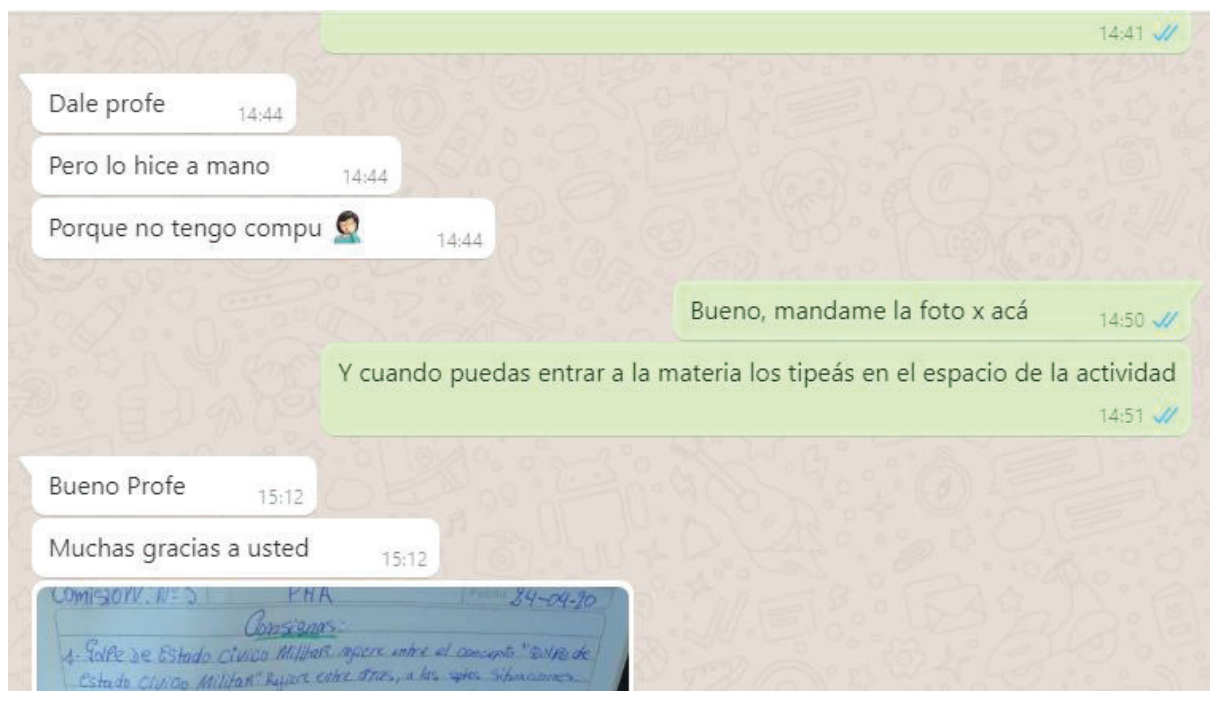

Mantenernos comunicados por diversos canales también pone en evidencia las brechas que existen entre los estudiantes, esto nos obliga a posicionarnos desde la mirada ética y actuar en consecuencia para garantizar el sostén de las trayectorias.

Figura 2: Ejemplo de las "capturas de pantalla" enviada por una docente, acompañada de la explicación sobre el sentido de esta.

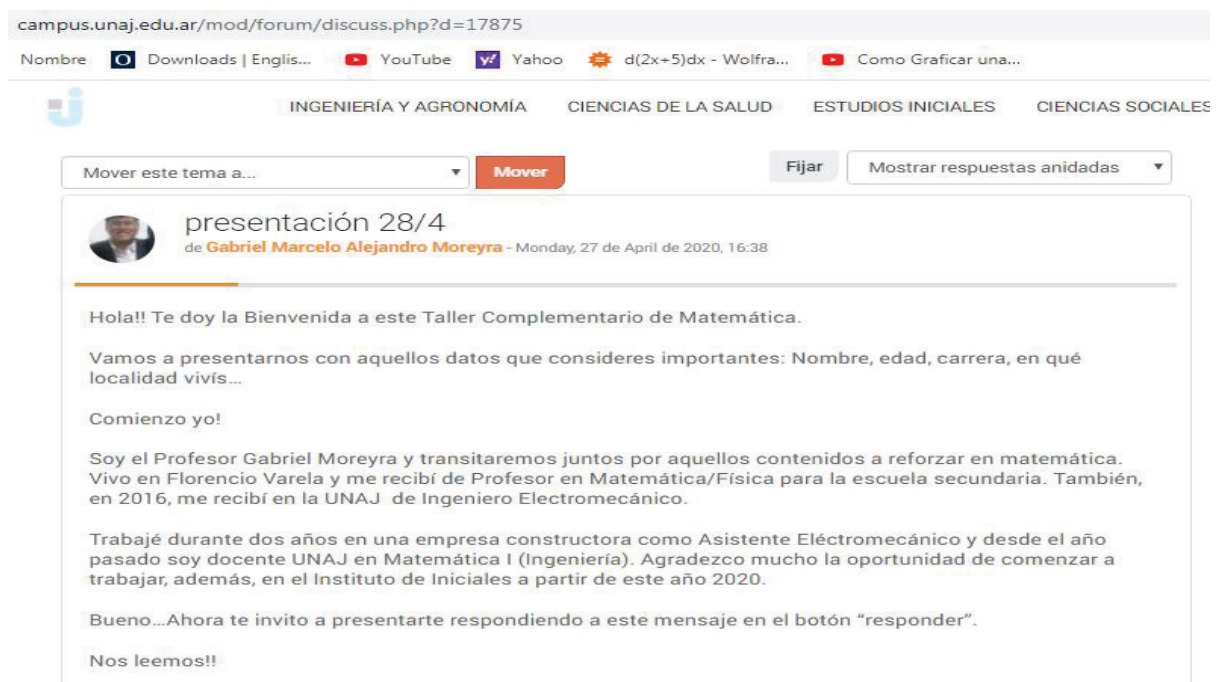

Primera captura: lenguaje positivo, ante una situación adversa como el aislamiento, muchos de los estudiantes, tienen que ir despacio ante la plataforma...si hay algo obvio para mí, como el indicar "hace click" debo pensar que del otro lado hay alguien que está con un celular y "presiona el botón responder" suele ser más efectivo.

El "nos vemos", cambia de a poco a un "nos leemos" y otra cosa: mi tiempo en línea, no es necesariamente igual al de aquel que está del otro lado. Cortes de luz o Internet, se convierten en causas de atemporalidad.

Figura 3: Otro ejemplo de las "capturas de pantalla" enviada por un docente, acompañado de la explicación sobre el sentido de esta. 
Tenemos la hipótesis que el trabajo solicitado exigía esfuerzo y dedicación, que, en este contexto, se pudo haber transformado en una exigencia más. En virtud de ello, para poder continuar la investigación, durante el siguiente cuatrimestre, decidimos retomar el contacto proponiendo realizar algunas entrevistas, no solamente a quienes nos habían enviado las capturas, sino también al grupo original de 50 docentes comenzado el segundo cuatrimestre. Para nuestra sorpresa, cerca de 20 de ellos se mostraron nuevamente interesados en ser entrevistados. Tenemos la hipótesis de que, más allá del menor compromiso que podría exigir participar de una entrevista, en realidad, se trataba ya con un recorrido realizado en esta virtualidad forzada de un espacio de "escucha" que colegas de la misma institución le ofrecían para compartir la experiencia. De hecho, en todas las entrevistas, de una u otra manera, agradecieron esta posibilidad.

Finalmente, 17 de ellos participaron de los grupos que conformamos para las entrevistas grupales. En este texto, daremos cuenta específicamente de parte de la 2 da fase denominada de contraste. Los datos emergen de una serie de entrevistas grupales realizadas durante el 2do cuatrimestre del 2020 que han sido grabadas y aquí presentaremos algunos extractos. En general, se han organizado seis grupos con dos o tres integrantes de las diferentes materias que componen el Ciclo Inicial.

El proceso de codificación fue doble: en primer lugar, a partir de las categorías y sus propiedades que sirvieron de ejes para elaborar las preguntas de las entrevistas (Bifano, Almirón, Cabaña, Gonzalez, 2020); $y$, en segundo lugar, a partir de las notas emergidas de las lecturas de las entrevistas sobre la base de la identificación de incidentes particulares. Cada investigador/a desgrabó y analizó alguna de las entrevistas que no realizó y compartió sus notas con los demás investigadores en un espacio común.

\section{ANÁLISIS DE LOS DATOS}

De las tres categorías inicialmente consideradas (clase, estudiantes y recursos), en este artículo daremos cuenta de la denominada clase, alguna de sus propiedades, su necesaria resignificación como categoría, y sus relaciones con otros emergentes revelados en el análisis de cuatro entrevistas grupales.

\section{Categoría: clase}

Esta es una de las más relevantes de nuestra investigación pues si bien en el contexto presencial habitual es posible caracterizar con cierta naturalidad en qué consiste una clase y cuáles son sus límites espacio-temporales; a partir de las expectativas acerca de qué 
elementos constituyen la misma, en el contexto de esta virtualidad forzada, hemos considerado fundamental reconstruir su significado a partir de la mirada de sus protagonistas. En este texto, solo presentaremos los datos recogidos de las entrevistas a las y los docentes, con lo cual, las voces de las y los estudiantes, cuando emergen, son a través de la "interpretación" que hacen sus propios docentes.

\section{En este texto, solo presentaremos los datos recogidos de las entrevistas a las y los docentes, con lo cual, las voces de las y los estudiantes, cuando emergen, son a través de la "interpre- tación" que hacen sus propios docentes.}

En general, como ya habíamos podido ver por las "capturas de pantallas" que nos habían enviado, todas las materias organizaron un material de base con explicaciones escritas, videos, ejercicios con software (en el caso de Ml) que ponían a disposición de las y los estudiantes semana a semana. En parte, podríamos decir, esto ya constituye "una clase" o al menos, parte de ella. Luego, cada docente administraba los foros de debate en torno a estas lecturas y/o a los ejercicios que se proponían. Esto posiblemente formatea de alguna manera el trabajo docente a partir de esta forma de organizar institucionalmente la tarea. Así surge la pregunta: ¿qué es una clase en este contexto?

Si bien en algunas entrevistas hicimos la pregunta explícitamente, en otras esa cuestión emergió espontáneamente de las y los entrevistados cuando comentaban los desafíos a los que se tenían que enfrentar. En ese sentido, podemos encontrar diversas intervenciones que nos permiten delinear cuál es la idea de clase que tienen las y los docentes:

«No existe más ese concepto, no hay más "la clase". Por una cuestión más personal me gusta organizar, como tener una especie de rutina. Como doy clases los lunes y jueves, los lunes les mando mensajes por todas las vías (foros, mail, WhatsApp): "Hola, comenzamos la tercera semana"y les recuerdo las tareas y les pauto (por ahí soy un poco cuadrada) los encuentros sincrónicos si es que hay». (Florencia, 6 años de antigüedad en UNAJ, docente de TLE).

«Una clase extendida. Una clase que tiene múltiples espacios, múltiples lugares, múltiples mediadores... Uno tiene que organizarse y en ese sentido la materia está organizada, las clases comienzan los martes y la entrega de los trabajos es el lunes anterior a que se publique la otra clase. Durante ese período vamos manejando los distintos espacios de la clase. [...] En resumen, la clase, si tengo que decir algo cronológico, dura una semana, es una clase extendida. Una clase que en realidad son microclases». (Pamela, 1 año de antigüedad en UNAJ, docente de PHA). 
Otro de los elementos que emergen de esta coyuntura tiene que ver con los aspectos inherentes a lo espacio-temporal de las clases, es decir, su duración en el tiempo y los "contornos" espaciales que la delimitan. Resulta evidente que, en este contexto, a la decisión de hacer una propuesta asincrónica y sus implicancias respecto de los límites de duración de una clase, se le suma el hecho de que ya no están las paredes del aula que configuran no solamente lo espacial sino también lo público y privado del desarrollo de una clase. Estos elementos, se ilustran con algunos extractos bajo las propiedades que presentamos seguidamente.

\section{Resulta evidente que, en este contexto, a la decisión de hacer una propuesta asincrónica y sus implicancias respecto de los límites de duración de una clase, se le suma el hecho de que ya no están las paredes del aula que configuran no solamente lo espacial sino también lo público y privado del desarrollo de una clase.}

Propiedad: inicio, fin, duración de una clase

«Los límites de la clase se perdieron. A mí la cursada virtual me genera... siempre estoy en una sensación de falta personal, de que no llego, de que no alcanzo, las horas que estoy son muchísimas. [...] Entro a la mañana, a la tarde, a la noche. Esto me ha generado un estado de ansiedad grave. [...] La clase se desdibujó y se extendió demasiadas horas también». (Soledad, 6 años de antigüedad en UNAJ, docente de MI).

«En mi caso, tenías ciertos horarios...cumplíamos ciertos "ritos". Las preguntas se hacían en los horarios de clase o en los días que tenía clase... lo que de algún modo mostraba que había un comienzo es que había un pequeño foro». (Verónica, 10 años de antigüedad en UNAJ, docente de TLE).

Propiedad: carácter de una clase, público vs. privado.

«El trabajo se metió en casa. Es difícil separar la vida laboral de la vida privada». (Gabriela, 10 años de antigüedad en UNAJ, docente de TLE).

«Dar clase ya no es más privado (pueden aparecer mi niña, hay una cama porque me estoy mudando, el albañil...)». (Zulema, 4 años de antigüedad en la UNAJ, docente de MI). 
Esta propiedad no fue inicialmente prevista en la primera fase y ha emergido con fuerza, a veces, desde lugares disímiles:

«Angustia más que nada. [...] No soy de la virtualidad. Angustia por los estudiantes que fueron dejando y una no sabe por qué. [...] como resumen diría una gran angustia al principio provocada por la incertidumbre y un fuerte "no quiero". Nadie me enseñó esto.» (Gabriela, 10 años de antigüedad en UNAJ, docente de TLE).

«[...] me sentía sola, no sabía si estaban o no los estudiantes del otro lado.» (Victoria, 10 años de antigüedad en UNAJ, docente de PHA).

Estas son algunas de las intervenciones que recopilamos y que dan cuenta de que el concepto de clase se modifica dentro de la virtualidad, aunque todavía no podamos definir claramente qué implica el nuevo concepto. Una de las cuestiones emergentes ha sido los difusos límites para encerrar a una clase virtual tanto en lo que hace a la temporalidad como, en cierta manera, a lo espacial y la pertenencia o no a la esfera de lo público o lo privado, y los sentimientos encontrados que genera.

\section{CONCLUSIONES}

Si bien la investigación aún está en proceso de desarrollo, es posible llegar a delinear algunas conclusiones respecto de los análisis aquí efectuados.

Ante la pregunta en qué consiste una clase virtual, las definiciones que surgen se relacionan con el tipo de actividad que desarrollan, los espacios de intercambio que proponen y sobre todo a los tiempos que implican. Hay coincidencia en que la temporalidad se transforma en relación con la clase presencial: hay quien expresa que se vuelven muchas clases dentro de una, como también quien directamente no puede definirlo. Parecen tener claro cuándo ocurre el comienzo de la clase: lo vinculan con el momento en que se publican nuevos contenidos, textos o actividades, pero en general no pueden explicitar cuándo termina. Observamos también que los márgenes de la clase se vuelven difusos vinculados a que el trabajo desde las casas no permite separar tan fácilmente la vida personal de la vida profesional.

Ante la pregunta en qué consiste una clase virtual, las definiciones que surgen se relacionan con el tipo de actividad que desarrollan, los espacios de intercambio que proponen y sobre todo a los tiempos que implican. Hay coincidencia en que la temporalidad se transforma en relación con la clase presencial: hay quien expresa que se vuelven muchas clases dentro de una, como también quien directamente no puede definirlo. 
En línea con los sentimientos que despierta este cambio de escenario en la educación, nos pareció relevante la necesidad de las y los docentes de ser escuchadas/os, de participar de espacios de intercambio y reflexión una vez transitados cierto tiempo en esta nueva modalidad.

Nos resulta importante destacar que asociado al concepto de clase emergen otras propiedades no previstas inicialmente, como los sentimientos y sensaciones que genera este escenario virtual, así como a la vez se ve íntimamente ligada a los recursos docentes y a las relaciones que establecen con sus estudiantes, categorías que también hemos investigado pero que daremos cuenta en futuras publicaciones.

Para concluir, nos parece insoslayable abrir la reflexión sobre cuál será el impacto de esta experiencia en la vuelta a una cierta nueva normalidad, cuáles serán las lecciones aprendidas, cómo se reconfigurarán las clases presenciales, cuáles de los recursos producidos realmente seguirán teniendo vigencia e integrados a la práctica, entre otros interrogantes que aún permanecen abiertos. 


\section{BIBLIOGRAFÍA}

Bifano, F.; Almirón, A.: Cabaña, L.; Gonzalez, K. (2020). "Clases virtuales en el ingreso universitario: la emergencia en la construcción de sentido". Trayectorias Universitarias, 6(10), 022. https://doi.org/10.24215/24690090e022

Glaser, B., \& Strauss, A. (1999).“'Discovery of GroundedTheory:Strategies forQualitative Research" (1st ed.). Routledge. https://doi.org/10.4324/9780203793206

Gueudet, G. \& Trouche, L. (2009). "Towards new documentation systems for mathematics teachers?" Educational Studies in Mathematics, 71(3), 199-218. https://doi.org/10.1007/s10649-008-9159-8

Lora A. A., Cavadias, L. M. y Miranda, A. J. (2017). “La Teoría Fundamentada en el Marco de la Investigación Educativa". Saber, ciencia y libertad, 12(1), 10. https://doi.org/10.18041/2382-3240/saber.2017v12n1.1475.

Orozco, J. (2013). “La Virtualidad en Educación Superior: Una Perspectiva”. XIV Encuentro Internacional Virtual Educa. Colombia. 